\title{
GASTROPLASTIA REDUTORA COM BYPASS GASTROJEJUNAL EM Y-DE-ROUX: conversão para bypass gastrointestinal distal por perda insuficiente de peso - experiência em 41 pacientes
}

\author{
José Carlos PAREJA ${ }^{1,2}$, Victor Fernando PILLA ${ }^{2}$, Francisco CALLEJAS-NETO ${ }^{1,2}$, \\ João de Souza COELHO-NETO ${ }^{2}$, Elinton Adami CHAIM $^{1}$ e Daniéla Oliveira MAGRO²
}

RESUMO - Racional - O tratamento cirúrgico é a única modalidade efetiva de tratamento da obesidade mórbida. O insucesso do bypass gastrointestinal (percentagem de perda de excesso de peso inferior a 50\%) pode chegar a $10 \%$ dos pacientes operados a longo prazo (acima de 5, num ideal de 10 anos). Objetivos - Avaliar os resultados, em termos de perda de peso, dos pacientes submetidos a reoperação com a finalidade de aumentar o componente disabsortivo. Casuística e Métodos - Estudaram-se 41 doentes, sendo 32 submetidos a reoperação por uma de três técnicas cirúrgicas (Fobi, Brolin e bypass gastrojejunoileal distal) nas quais foi realizada diminuição da área absortiva do intestino delgado. Resultados - Os doentes submetidos a bypass gastrojejunoileal distal apresentaram resultados superiores aos demais $(69,7 \%)$. Conclusão - O emprego do bypass distal pode ser utilizado em casos selecionados com o intuito de melhorar os resultados em termos de perda de peso. É aconselhável a centralização desses procedimentos em serviços de referência com experiência na área específica de cirurgia bariátrica, para acompanhamento rigoroso desses doentes.

DESCRITORES - Obesidade mórbida, cirurgia. Gastroplastia. Derivação gástrica. Derivação em Y-de-Roux.

\section{INTRODUÇÃO}

A obesidade severa é uma doença crônica, evolutiva, com co-morbidades médicas, sociais, econômicas e psicológicas. A cirurgia é o único procedimento que resulta em perda de peso significativa e permanente nesses doentes, com melhora das condições das referidas co-morbidades. O bypass gastrojejunal em Y-de-Roux, que produz restrição alimentar e má absorção relativa, é considerada a cirurgia mais eficaz nessa proposição, sendo a mais utilizada nos Estados Unidos da América, país em que é realizado o maior número de cirurgias no mundo e, também, em nosso meio ${ }^{(8,11)}$. Há variações na técnica de realização dessa cirurgia, no tipo de reservatório gástrico (horizontal ou vertical), na colocação ou não de um anteparo inelástico (Silastic ou Marlex) na bolsa gástrica, tentando diminuir a velocidade do esvaziamento gástrico e aumentar o tempo de saciedade, e em relação à medida do comprimento das alças aferente (bíliopancreática) e eferente (alimentar). $\mathrm{O}$ índice de falência (percentagem de perda de excesso de peso inferior a 50\%) desse tipo de procedimento varia na literatura, com Fobi $3 \%$ a $5 \%$ ), Capella (7\%), Mac Lean (7\%), chegando a $33 \%$ em superobesos na série de Brolin ${ }^{(3,4,6)}$.

Nos casos em que haja insucesso, a proposição cirúrgica de resgate mais utilizada é a realização do bypass gastrojejunoileal distal ou gastroileal distal, também denominado de derivação bíliopancreática distal com bypass gastrointestinal ${ }^{(5,6,9,13)}$.

\section{PACIENTES E MÉTODOS}

Analisaram-se retrospectivamente os prontuários de 41 doentes submetidos a alguma forma de bypass gastrointestinal distal, que foram divididos em dois grupos: o primeiro com 9 doentes nos quais foi realizado o bypass distal como procedimento inicial e o segundo com 32 doentes nos quais foi realizada a conversão de um bypass gastrojejunal tipo Fobi-Capella em um bypass distal por falência na perda de peso.

${ }^{1}$ Grupo de Cirurgia de Obesidade, Disciplina de Moléstias do Aparelho Digestivo, Faculdade de Ciências Médicas da Universidade Estadual de Campinas; ${ }^{2}$ Centro de Cirurgia de Obesidade de Campinas, Campinas, SP.

Endereço para correspondência: Dr. José Carlos Pareja - Av. Heitor Penteado, 522 - Taquaral - 13075-460 - Campinas, SP. E-mail: jcpareja@obesidadesevera.com.br 


\section{Técnica cirúrgica}

Os procedimentos cirúrgicos denominados de bypass distal, ou derivação bíliopancreática distal com bypass gastroejunal ou gastroileal podem ser divididos conforme as proposições de Fobi, Brolin ou a gastrojejunoileal distal (tipo Scopinaro) proposta em nosso meio por Marchesini ${ }^{(9)}$.

Gastroplastia com bypass gastrojejunal distal tipo Fobi

Nesse método, após a cirurgia do bypass gastrojejunal inicial proposta por esse autor, em que a alça aferente tem $30 \mathrm{~cm}$ e a alça alimentar $90 \mathrm{~cm}$, é desfeita a êntero-êntero anastomose, a seguir refeita distalmente na metade do comprimento do intestino delgado, que é novamente medido a partir do ângulo de Treitz. Dessa forma, o canal comum e o canal alimentar ficam com 3 a $3,5 \mathrm{~m}$, permanecendo este último com $90 \mathrm{~cm}$.

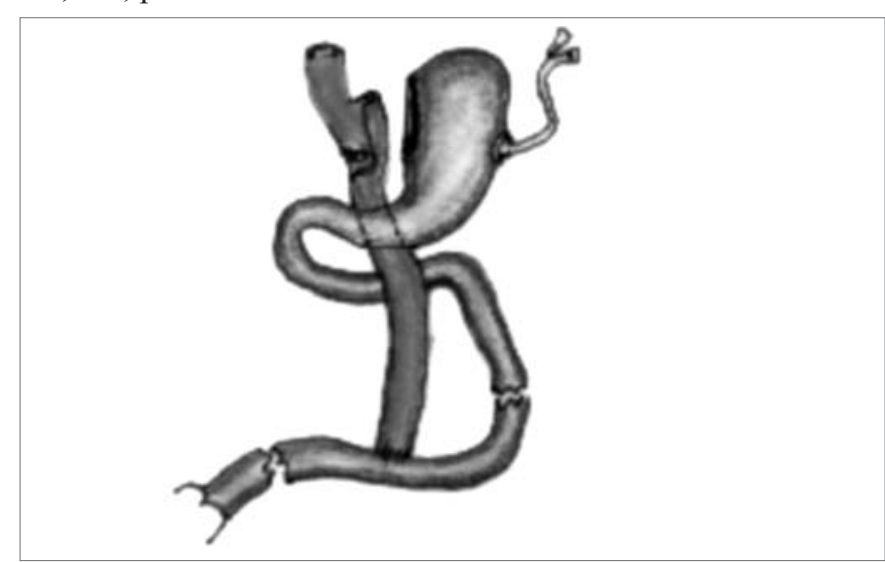

FIGURA 1 - Bypass gastrojejunal distal tipo Fobi

Gastroplastia com bypass gastrojejunal distal tipo Brolin

Nessa técnica, após a cirurgia do bypass gastrojejunal inicial em que a alça aferente tem $30 \mathrm{~cm}$ e a alça alimentar $75 \mathrm{~cm}$, é desfeita a êntero-êntero anastomose, que é refeita distalmente, a $75 \mathrm{~cm}$ da válvula íleocecal, ficando, então, o canal comum com $75 \mathrm{~cm}$ de comprimento, e além da alça biliopancreática excluída, com $30 \mathrm{~cm}$, o restante do intestino delgado permanece como canal alimentar. Aqui também a soma do canal alimentar e comum é, geralmente, maior do que $350 \mathrm{~cm}$.

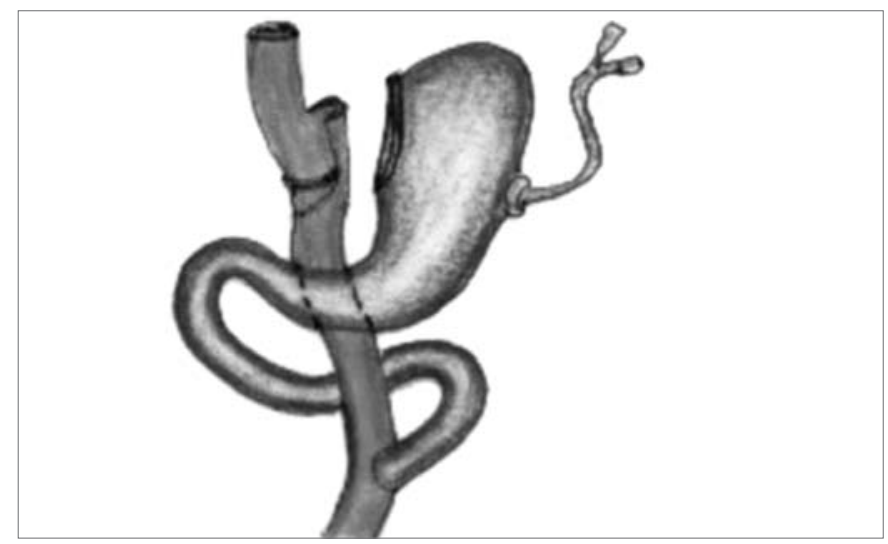

FIGURA 2 - Bypass gastrojejunal distal tipo Brolin
Bypass distal gastrojejunoileal distal (tipo Scopinaro)

Nesse procedimento proposto em nosso meio por MARCHESINI et al. ${ }^{(9)}$, é desfeita a êntero-êntero anastomose que é refeita com alça aferente jejunal e a alça ileal a $100 \mathrm{~cm}$ da válvula ileocecal. Faz-se uma ressecção da parte jejunal da alça alimentar, é refeita uma êntero-êntero anastomose entre o íleo $(220 \mathrm{~cm})$ e o restante da alça jejunal, em média $20 \mathrm{~cm}$, que está anastomosada à bolsa gástrica. Dessa forma, tem-se um canal alimentar composto em média de $20 \mathrm{~cm}$ de jejuno e $220-230 \mathrm{~cm}$ de íleo. O canal alimentar é composto praticamente de íleo, à semelhança de técnica de Scopinaro e o canal comum permanece com $100 \mathrm{~cm}$.

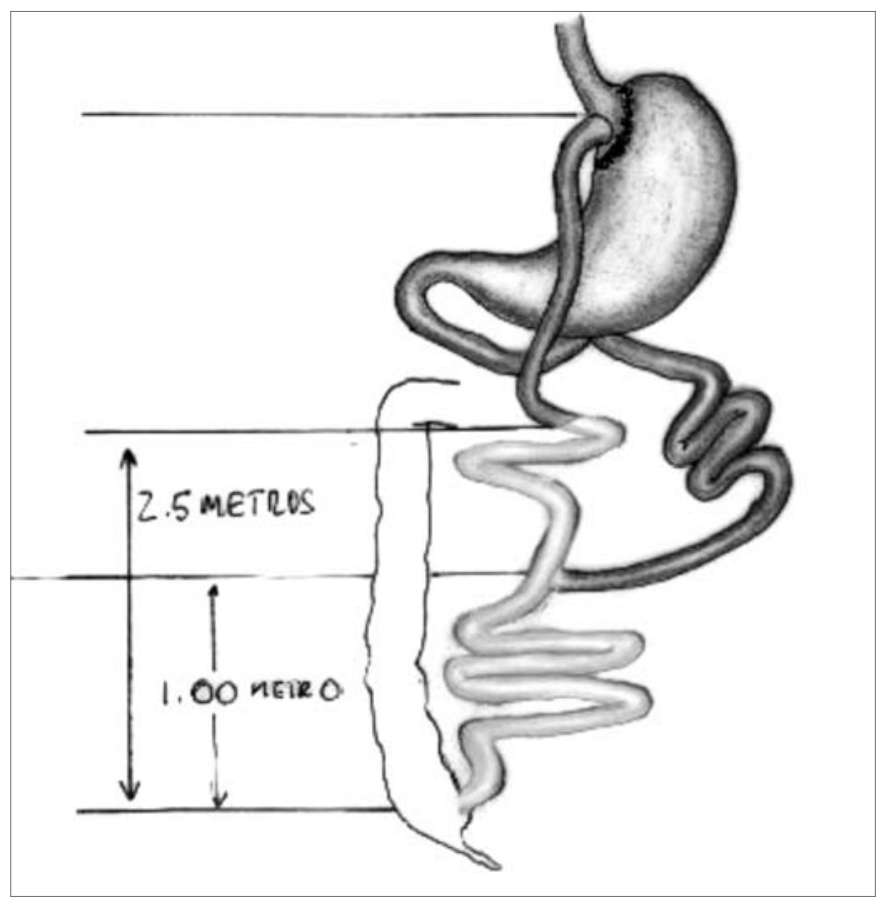

FIGURA 3 - Bypass gastrojejunoileal distal (tipo Scopinaro)

Grupo I (bypass distal como primeiro procedimento) (Tabela 1)

Neste grupo foram incluídos os doentes nos quais foi realizado o bypass distal como primeira cirurgia, pois se tratava de pacientes superobesos. Nas duas técnicas cirúrgicas (Fobi e Brolin) são utilizadas alças jejunais de maior comprimento com o intuito de diminuir a capacidade de absorção intestinal.

\section{Grupo II}

As características dos doentes que foram submetidos ao bypass gastrojejunoileal distal ou gastroileal distal podem ser vistas na Tabela 2.

Como pode ser visto na Tabela 2 , os doentes operados pela técnica de Fobi, haviam perdido, em média, $62,38 \%$ do excesso de peso (portanto acima dos $50 \%$ definidos como falência), mas não estavam satisfeitos com o peso atual e apresentavam tendência a aumento do mesmo; todos eles tinham indicação de outros procedimentos a serem feitos associadamente, como herniorrafia incisional ou colecistectomia. 
Pareja JC, Pilla VF, Callejas-Neto F, Coelho-Neto JS, Chaim EA, Magro DO. Gastroplastia redutora com bypass gastrojejunal em Y-de-Roux: conversão para bypass gastrointestinal distal por perda insuficiente de peso - experiência em 41 pacientes

TABELA 1 - Características dos doentes submetidos a bypass distal como primeiro procedimento bariátrico

\begin{tabular}{lcc}
\hline Bypass distal (1'a cirurgia) & Fobi & Brolin \\
\hline Número de doentes & 3 & 6 \\
Sexo M/F & $1 \mathrm{M} / 2 \mathrm{~F}$ & $1 \mathrm{M} / 5 \mathrm{~F}$ \\
Idade média (anos) & $41(40-42)$ & $42,1(31-50)$ \\
Peso médio (kg) & $147(112,5-186,4)$ & $171,43(136,8-220,0)$ \\
IMC médio inicial & $55,71(51,36-59,52)$ & $64,27(52,78-73,51)$ \\
\hline
\end{tabular}

$\mathrm{M}$ - masculino; $\mathrm{F}$ - feminino; $\mathrm{kg}$ - quilogramas; IMC - índice de massa corpórea

TABELA 2 - Características dos doentes submetidos a bypass distal como segundo procedimento bariátrico, por falência da perda de peso após a cirurgia convencional (Fobi-Capella)

\begin{tabular}{|c|c|c|c|c|}
\hline Bypass distal ( $2^{a}$ Cirurgia) & & BGJID & Brolin & Fobi \\
\hline Número & & 16 & 9 & 7 \\
\hline Sexo M-F & & $3 \mathrm{M} / 13 \mathrm{~F}$ & $3 \mathrm{M} / 6 \mathrm{~F}$ & $2 \mathrm{M} / 5 \mathrm{~F}$ \\
\hline Idade média (anos) & & $36,18(20-45)$ & $44,55(32-53)$ & $42,85(32-55)$ \\
\hline Peso médio (kg) & $1^{\text {a }}$ cirurgia & $152,31(94-220)$ & $135,68(94-186)$ & $142,88(106-188)$ \\
\hline IMC médio & $1^{\text {a }}$ cirurgia & $56,15(43,66-70,83)$ & $49,3(36,05-72,66)$ & $51,9(41,41-63,55)$ \\
\hline Perda excesso de peso $(\%)$ & após a $1^{a}$ cirurgia & $49,3 \%(28,8-64,5)$ & $43,7 \%(20-59,7)$ & $62,38 \%(54,6-91)$ \\
\hline Follow-up (meses) & após a $1^{\mathrm{a}}$ cirurgia & $27,8 \mathrm{~m}(15-46)$ & $17,6 \mathrm{~m}(3-30)$ & $17,42 \mathrm{~m}(8-27)$ \\
\hline
\end{tabular}

M - masculino; F- feminino; kg - quilogramas; m - meses; IMC - índice de massa corpórea; BGJID - bypass gastrojejunoileal distal

\section{RESULTADOS}

Pode-se ver na Tabela 3 , que dos doentes operados pela técnica do bypass gastrojejunoileal distal (BGJID), cerca de $62,5 \%$ eram superobesos inicialmente. No grupo Brolin representavam $33,53 \%$ e no grupo Fobi $42,8 \%$. Verificou-se queda do IMC proporcionalmente maior no grupo de doentes submetidos ao bypass gastrojejunoileal distal (tipo Scopinaro).

$\mathrm{Na}$ análise da Tabela 3 vê-se que a percentagem final de perda do excesso de peso foi de $69,7 \%$ nos doentes submetidos a BGJID, de $65,05 \%$ nos submetidos ao procedimento tipo Brolin e de $74,84 \%$ nos submetidos a técnica Fobi. Não há como fazer comparação estatística pelo pequeno número de pacientes em cada grupo, e porque os doentes do grupo Fobi foram operados na revisão cirúrgica com IMC menor de que os doentes dos dois outros grupos.

\section{DISCUSSÃO}

A obesidade severa é uma doença não responsiva a tratamento clínico, mas pode ser abordada cirurgicamente, o que permite melhores condições de acompanhamento e controle de parâmetros de saúde a longo prazo. Em virtude da multiplicidade de fatores que atuam na obesidade, não existe nenhum tipo de cirurgia totalmente efetivo para todos os doentes ${ }^{(11)}$. A gastroplastia com bypass gastrojejunal com o uso de anel inelástico (Silastic ou Marlex) na bolsa gástrica, produz restrição mais efetiva, sendo a opção cirúrgica por cerca de $30 \%$ dos cirurgiões dos EUA, entre os quais se destacam Fobi, Capella, e em nosso meio divulgada por Garrido e realizada por um grande número de cirurgiões, entre os quais os autores deste estudo. ${ }^{(1,4,6,8,10)}$

A pequena disseminação das cirurgias disabsortivas (tipo Scopinaro ou duodenal switch) nos EUA pode ser explicada pelo fato destas técnicas apresentarem maior índice de complicações metabólicas a médio e longo prazos, cujo estigma estreitamente relacionado ao bypass jejunoileal motivou muito do desprestígio e ceticismo da cirurgia bariátrica nesse país ${ }^{(2,7)}$. Porém, a técnica denominada duodenal switch, por apresentar teoricamente, menos complicações metabólicas do que a técnica de Scopinaro, tem sido utilizada por alguns cirurgiões norte-americanos. Deve-se lembrar, também, que

TABELA 3 - Resultados obtidos pelos três métodos cirúrgicos, utilizados como segundo procedimento cirúrgico na falência da perda de peso após a gastroplastia com bypass gastrojejunal tipo Fobi-Capella

\begin{tabular}{|c|c|c|c|c|}
\hline Bypass distal ( 2 a cirurgia) & & BGJID & Brolin & Fobi \\
\hline Peso médio (kg) & $2^{\mathrm{a}}$ cirurgia & $110,53(75,6-166)$ & $105,2(88,8-123,2)$ & $97,31(82-120)$ \\
\hline IMC médio & $2^{\mathrm{a}}$ cirurgia & $40,76(31,6-55,5)$ & $38,5(33,1-48,1)$ & $35,52(26,5-41,4)$ \\
\hline IMC médio & Final & $34,88(29,4-48,2)$ & $34,57(28-44,2)$ & $32,18(25,1-38,1)$ \\
\hline Perda de excesso de peso (\%) & Após $2^{a}$ cirurgia & $69,7 \%(54,6-88,2 \%)$ & $65,05 \%(52,7 \%-86,6 \%)$ & $74,84 \% \%(61,1-99,4 \%)$ \\
\hline Variação IMC & & $6,52(2,3-18,7)$ & $4,35(0-8,3)$ & $3,34(1,4-5,7)$ \\
\hline Seguimento & Após $2^{\mathrm{a}}$ cirurgia & $11,31 \mathrm{~m}(5-19 \mathrm{~m})$ & $16 \mathrm{~m}(3-28 \mathrm{~m})$ & $19,5 \mathrm{~m}(2,5-28 \mathrm{~m})$ \\
\hline Obs & & 10/16 superobesos & $3 / 9$ superobesos & $3 / 7$ superobesos \\
\hline
\end{tabular}

M-masculino F-feminino kg - quilogramas m - meses; IMC - Índice de massa corpórea; BGJID - Bypass gastrojejunoileal distal 
a estrutura do atendimento médico norte-americano baseia-se no pagamento a cada consulta/procedimento, com eventual reembolso pela seguradora de saúde (para equipe cirúrgica em geral extingue-se a cobertura 90 dias após a cirurgia), ao contrário do sistema de saúde em países como a Itália, Canadá, Espanha, Austrália em que o atendimento médico é subvencionado pelo governo ${ }^{(5)}$.

FOBI et al. ${ }^{(6)}$, em 2.391 operações desse tipo, teve um índice de insucesso de 3\% (65 pacientes) em 5 anos de seguimento. Esses pacientes foram submetidos a uma cirurgia de revisão, denominada da gastroplastia com bypass gastrojejunal distal, conforme mostrado previamente. CAPELLA e CAPELLA ${ }^{(4)}$ chamam atenção que MacLean, em sua série de doentes operados sem anel inelástico no bypass clássico, teve falência em $7 \%$ dos doentes no seguimento entre 3 a 5 anos. Já no grupo de superobeso o índice de falha elevou-se para $43 \%$ dos doentes. CAPELLA e CAPELLA ${ }^{(4)}$ tiveram uma falência na perda de peso na sua série global em $7 \%$ dos doentes e isoladamente nos superobesos em apenas 3\% doentes. A perda de peso na série desse autor em superobesos foi menor de que a de Marceau com derivação biliopancreática (tipo duodenal switch) por ele empregada, com perda de excesso de peso menor que $50 \%$ em $13 \%$ dos doentes.

BIRON et al. ${ }^{(2)}$ (do grupo de Marceau) utilizam, ainda, como critério para avaliação de sucesso em cirurgia bariátrica o IMC de 35 para obesos mórbidos e 40 para superobesos. Na análise de seus resultados os doentes foram estratificados por IMC inicial e de acordo com o tempo de seguimento. Este apresentou taxa de seguimento de $99 \%$, com duração média de 7,9 anos. Ao cabo de 10 anos, $20 \%$ dos obesos mórbidos permaneciam com IMC superior a 35\% e $40 \%$ dos superobesos apresentavam um IMC maior do que 40.

Com o uso do bypass clássico (sem anel), BROLIN et al. ${ }^{(3)}$ em uma série com superobesos, tiveram como resultado uma perda de excesso de peso menor do que $50 \%$ em $33 \%$ dos casos após 5 anos.

Na presente série, utilizou-se como procedimento inicial o bypass gastrojejunal distal pela técnica de Fobi em três doentes, com perda de excesso de peso média de $79 \%$, e em seis doentes utilizou-se a técnica de bypass proposta por Brolin, com perda média de excesso de peso de $77,46 \%$, com IMC final de 34,48 (média) com 17 meses de seguimento. Todos os doentes eram superobesos (IMC $>50$ ). Embora não indicado na falha de uma cirurgia prévia, inclui-se este grupo para análise e comparação de resultados.

O uso do bypass distal como cirurgia de revisão da falência da perda de peso foi utilizada em $32(3,4 \%)$ de 929 doentes inicialmente operados pelo grupo, autores desta casuística, pela técnica de Fobi-Capella. Porém, considerando os doentes ainda não operados e que perderam menos do que $50 \%$ do excesso de peso, o insucesso da cirurgia inicial quanto à perda de peso foi em $6 \%$ dos casos. Quando se comparou a perda de peso na segunda cirurgia (Tabela 2), viu-se que a variação do IMC foi de 6,52 (em 16 casos) pela técnica do BGJID, dos quais 10 ainda eram superobesos, com IMC final de 34,88 , após 11,3 meses de seguimento. Já na técnica preconizada por Brolin, em nove casos, dos quais três eram superobesos, a variação do IMC foi de 4,35 e o IMC final foi de 34,57. Na técnica preconizada por Fobi, a variação do IMC foi 3,34, com IMC final 32,18 .

Por esses dados, embora tivessem sido empregados três procedimentos diferentes, parece que a técnica do bypass gastrojejunoileal distal tipo Scopinaro, onde o canal alimentar tem o íleo como componente principal e o canal comum tem $100 \mathrm{~cm}$, foi o procedimento mais efetivo quanto à perda de peso. Isto está de acordo com os conceitos de TORRES e $\mathrm{OCA}^{(12)}$, e TORRES ${ }^{(13)}$, de que quando o canal comum e o canal alimentar são maiores de que $360 \mathrm{~cm}$, os resultados de perda de peso a longo prazo são semelhantes aos obtidos com o bypass gastrojejunal clássico, utilizado na primeira cirurgia ${ }^{(12}$, 13). Nos procedimentos preconizados por Fobi e Brolin como cirurgia de revisão, o canal comum e o canal alimentar têm 360 $\mathrm{cm}$ ou mais em média o que, conforme parecer dos presentes autores, explica a menor efetividade dos resultados deste tipo de bypass distal ${ }^{(3,6)}$. Até o momento não houve problemas de diarréia ou desnutrição com o uso desse procedimento, diferentemente do que ocorreu com as séries de FOBI et al.(6) e TORRES ${ }^{(13)}$. Provavelmente isso se explica pela diferença do tipo de alimentação em nosso meio (pequena ingestão de gorduras pelos pacientes desta série, com predominância de carboidratos), quando comparados ao tipo de alimentação do paciente nos $\mathrm{EUA}^{(10)}$. Este fator poderá ter importância, ainda, em relação ao perfil do hábito intestinal (freqüência e consistência das evacuações) do grupo feminino (a maioria em termos de cirurgia bariátrica). Este fato pode explicar, em parte, os resultados inferiores no grupo feminino submetido as técnicas de alongamento da alça.

Nos doentes, cuja avaliação endoscópica demonstre que o anel está alargado, ou naqueles em que não foi colocado anel de contenção na cirurgia inicial, tem-se utilizado a colocação ou troca de anel por um mais ajustado. Esta manobra incrementa o papel restritivo da cirurgia, não devendo ser empregada em conjunto com a distalização da alça entérica. A casuística deste grupo ainda é pequena, mas tem tido resultados favoráveis com o reforço do componente restritivo neste grupo de doentes. BERTI e GARRIDO ${ }^{(1)}$ têm opinião semelhante, verificando melhor resultado com o emprego de reforço circunferencial ao reservatório gástrico.

Embora esta série tenha sido focada preferencialmente nas opções cirúrgicas nos casos de insucesso, deve-se ter em mente que na avaliação deste seleto grupo o perfil nutricional e psicológico deve ser revisado detalhadamente. Observou-se a importância da adequada aderência ao novo esquema de acompanhamento (dieta, consultas, exames), a necessidade de adequado nível cognitivo para se compreender os riscos e possíveis complicações e efeitos colaterais relacionados ao maior nível de disabsorção associado com uma restrição à livre alimentação. Naqueles doentes com distúrbios alimentares tipo "binge eating", que beliscam alimentos ricos em carboidratos e açúcar e que evacuam a cada dois ou três dias, em média, mesmo com a confecção de um bypass distal, talvez não se obtenha resultado satisfatório a longo prazo. 
A recomendação é que estes procedimentos revisionais sejam encaminhados aos grupos especializados (que apresentam grande volume de casos, bem como tempo prolongado de atuação na área) pois tratam-se de procedimentos de maior complexidade, sujeitos a maior morbimortalidade, bem como maior risco de questionamento jurídico.

\section{CONCLUSÃO}

O acompanhamento dos pacientes submetidos a cirurgia de revisão do bypass gastrointestinal aponta para melhor resultado em termos de perda de peso adicional, nos pacientes do sexo masculino submetidos ao bypass gastrojejunoileal distal tipo Scopinaro, em comparação àqueles submetidos a cirurgia de Brolin ou Fobi. No grupo feminino o aumento da porção disabsortiva não parece obter resultados semelhantes.

\section{CONCEITOS}

- Excesso de peso $(\mathrm{EP})=$ peso inicial - peso ideal

- Índice de massa corpórea $(\mathrm{IMC})=$ peso $\div$ altura $^{2}$

- Obesidade mórbida ou severa $=\mathrm{IMC} \geq$ a 40

- Peso ideal $(\mathrm{PI})=25 \times$ altura $^{2}$

- Perda de peso = peso inicial - peso atual

- Percentagem de perda de excesso de peso $(\% \mathrm{PEP})=$ perda de peso x $100 \div$ peso ideal

- Superobesidade $=\mathrm{IMC} \pm$ a 50

Pareja JC, Pilla VF, Callejas-Neto F, Coelho-Neto JS, Chaim EA, Magro DO. Gastric bypass Roux-en-Y gastrojejunostomy - conversion to distal gastrojejunoileostomy for weight loss failure - experience in 41 patients. Arq Gastroenterol 2005;42(4):196-200.

ABSTRACT - Background - Surgery is the only effective treatment for morbid obesity. Gastric bypass could fail in up to $10 \%$ of the patients (excess weight loss under 50\%). Aims - To evaluate the weight loss determined by reoperation performing disabsortive variation of gastric bypass. Patients and Methods - The records of 41 patients, in whom 32 were submitted to reoperation by one of three surgical techniques (Fobi, Brolin, distal gastrojejunoileal bypass) which consisted in increasing the disabsortive length of intestinal limb. Results - The patients submitted to distal gastrojejunoileal bypass showed the best results $(69,7 \%)$. Conclusion - The distal gastric bypass as a revisional procedure could be done in selected cases with the aim to improve the weight loss. It is advisable to refer these patients to selected centers (known as center of excellence) with experience in this area of bariatric surgery, in order to perform a very close follow-up.

HEADINGS - Obesity, morbid, surgery. Gastroplasty. Gastric bypass. Anastomosis, Roux-en-Y.

\section{REFERÊNCIAS BIBLIOGRÁFICAS}

1. Berti LV, Garrido Jr, AB. Bypass gástrico: é essencial a colocação de anel? In: SavassiRocha PR, editor. Obesidade e urgências gastroenterológicas. Rio de Janeiro: Medsi; 2003. p. $95-108$.

2. Biron S, Hould FS, Lebel S, Marceau S, Lescelleur O, Simard S, Marceau P. Twenty years of biliopancreatic diversion: what is the goal of the surgery? Obes Surg 2004; $14: 160-4$.

3. Brolin RE, Kenler HA, Gorman JH, Cody RP. Long limb gastric bypass in the superobese. A prospective randomized study. Ann Surg 1992;215:387-95.

4. Capella JF, Capella RF. The weight reduction operation choice: vertical banded gastroplasty or gastric bypass? Am J Surg 1996;171:74-9.

5. Cowan J Jr, Hiler ML, Martin LF. Reoperative surgery. In: Martin LF, editor. Obesity surgery. New York: McGraw-Hill; 2004. p.301-34.

6. Fobi MA, Lee H, Igwe D Jr., Felahy B, James E, Stanczyk M, Tambi J, Eyong P. Revision on failed gastric bypass to distal Roux-en-Y gastric bypass: a review of 65 cases. Obes Surg 2001;11:190-5.

7. Fox SR, Oh KH, Fox K. Vertical banded gastroplasty and distal gastric bypass as primary procedures: a comparison. Obes Surg 1996;6:412-25
8. Garrido Jr, AB. Cirurgia em obesos mórbidos: experiência pessoal. Arq Bras Endocrinol Metabol 2000,44:106-13.

9. Marchesini JB, Marchesini JC, Marchesini SD. "Scopinarização" - uma proposta para correção de operações bariátricas mal-sucedidas [resumo]. Anais do Congresso Brasileiro de Cirurgia Bariátrica, São Paulo, 1991.

10. Pareja JC, Pilla VF. Mecanismos de funcionamento da restrição gástrica, da derivação gastrojejunal e das derivações intestinais e biliopancreáticas. In: Garrido Jr AB, editor. Cirurgia da obesidade. São Paulo: Atheneu, 2003. p.129-34.

11. Pories WJ, Roth JS. Gastric bypass. In: Martin LF, editor. Obesity surgery. New York: McGraw-Hill; 2004. p.213-26.

12. Torres JC, Oca GF. Garrison RN. Gastric bypass Roux-en-Y gastrojejunostomy from the lesser curvature. South Med J 1983,76:1216-21.

13. Torres JC. Why I prefer gastric bypass distal Roux-en-Y gastroileostomy. Obes Surg 1991;1:189-94 